\title{
Transverse spin and transverse momentum distributions from COMPASS
}

\author{
Giulio Sbrizzai* \\ Trieste University and INFN \\ E-mail: giulio.sbrizzai@ts.infn.it
}

The study of the transverse-spin and transverse-momentum structure of the nucleon is part of the scientific program of COMPASS, a fixed-target experiment at the CERN SPS. By studying the azimuthal distributions of the hadrons produced in SIDIS off polarized and unpolarized nucleon targets one can get insight on the structure of the nucleon and on the possible correlations between transverse spin and intrinsic transverse momentum of the quarks. The results obtained for the Collins and Sivers asymmetries from the data collected in 2010 are here presented. They confirm the results obtained from 2007 with a reduction of more than a factor of two in the errors and the large statistics allows for a detailed investigation of the phase space covered by the spectrometer. New results obtained with a multi dimensional analysis of the unpolarized azimuthal asymmetries from the deuteron 2004 data are also presented.

Sixth International Conference on Quarks and Nuclear Physics,

April 16-20, 2012

Ecole Polytechnique, Palaiseau, Paris

\footnotetext{
* Speaker.
} 


\section{Introduction}

The investigation of the transverse spin and transverse momentum structure of the nucleon is one of the main topics of the COMPASS experiment. It is carried on by studying the azimuthal distributions of the hadrons produced in deep inelastic scattering (DIS) of $160 \mathrm{GeV} / \mathrm{c}$ positive muons off polarized and unpolarized nucleons. The high-momentum resolution of the COMPASS spectrometer [1], the large angular acceptance and the periodical reversal of the target polarization allow to measure the spin-dependent asymmetries with a great accuracy and to span a wide kinematical region. All the independent azimuthal modulations expected to appear in the SIDIS cross section can be measured at COMPASS from the same data. Their amplitudes are related both to the collinear Parton Distribution Functions (PDFs) and to the Transverse Momentum Dependent (TMD) PDFs describing the inner structure of the polarized nucleon.

In particular the Collins asymmetry [2]) shows up as the amplitude of the modulation of the sum of the azimuthal angle of the hadron $(\phi)$ and of the transverse spin $\left(\phi_{S}\right)$ around the virtual photon direction. It is given by the convolution of the transversity PDF, which gives the number density of the quarks polarized along the transverse polarization of the target nucleon minus the quarks which are oppositely polarized, with the Collins Fragmentation Function (FF), which is the quark transverse spin dependent part of the FF.

The Sivers asymmetry shows up as a $\phi-\phi_{S}$ modulation and it is given by the convolution of the unpolarized FF with the Sivers function, which gives the correlation between the transverse momentum of an unpolarized quark inside a transversely polarized nucleon and the transverse polarization of the nucleon [3]. Although it is naively T-odd, the Sivers function can in principle be different from zero thanks to the Final State Interactions [8]. Taking correctly into account the gauge links and the TMD formalism, the theory predicts that the Sivers asymmetries measured from a SIDIS reaction should have a opposite sign with respect to the one measured from a Drell-Yan reaction [9].

Complementary information on the nucleon structure, in particular on the relevance of the transverse momentum and higher-twist effects, is given by the measurements of the spin-averaged hadron azimuthal asymmetries. The $\cos \phi$ asymmetry is mainly given by the intrinsic transverse momentum of the quark and it is a kinematical effect (also known as "Cahn effect"). The TMD Boer-Mulders PDF, which correlates the quark transverse polarization and its intrinsic transverse momentum inside an unpolarized nucleon, appears at leading twist in the amplitude of the $\cos 2 \phi$ modulation. The same prediction on the change of sign from SIDIS to DY, as for the Sivers function, applies to the Boer-Mulders PDF [9]. The Cahn effect is also involved in this modulation, but at twist 4 , which means suppressed by a factor $1 / Q^{2}$. The $\sin \phi$ modulation is due only to higher twist effects and its amplitude is proportional to the beam polarization.

The results for the Collins and the Sivers asymmetries on the transversely polarized target, are discussed in sections 2. The first COMPASS results obtained on the spin averaged azimuthal asymmetries by means of a multi dimensional analysis are shown in section 3 .

\section{The Collins and Sivers Asymmetries}

The results on the Collins and Sivers asymmetries have been published for both transversely 


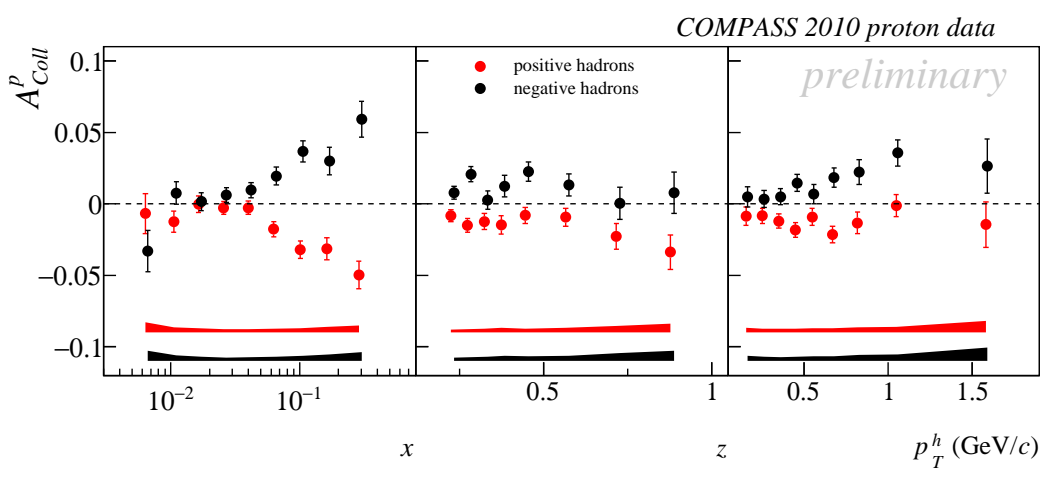

Figure 1: Collins asymmetry as function of $x, z$ and $P_{T}^{h}$, for positive and negative hadrons.

polarized deuteron [ [4] and protons [5] and new results for protons from data collected in 2010 were presented for the first time at Transversity 2011 []] and are described here.

The results for the Collins asymmetries from the 2010 COMPASS data taken on the transversely polarized proton are shown in fig. 1 1 as function of Bjorken scaling variable $x$, the fraction of the energy carried by the hadron $z$ and the transverse momentum of the hadron $P_{T}^{h}$. Both the results obtained for the positive hadrons (red points) and for the negative ones (black points) show a large signal in the valence region. The signal is of the same magnitude and opposite sign, which is negative for positive hadrons. The results obtained from the 2010 data nicely agree with the measurement performed on the 2007 transversely polarized proton data, but have a statistical error which is more than a factor of two smaller. The systematic error has been evaluated to be around $50 \%$ of the statistical error.

The Sivers asymmetries measured from the 2010 data are shown in fig. 2 as function of $x, z$ and $P_{T}^{h}$ for positive (red points) and negative (black points) hadrons. Again there is a good agreement with the published results from the 2007 run with a reduction of the statistical error of more than a factor of two. The asymmetry is definitely positive for positive hadrons and compatible with zero for negative hadrons. At variance with the Collins asymmetry, the Sivers asymmetry stays positive even at very small $x$, in the region of the sea.

The high statistic and the quality of the data allow to explore also different kinematical regions. The Sivers asymmetries extracted fro positive hadrons as function of $x, z$ and $P_{T}^{h}$ for small $y$ values $(0.05<y<0.1)$ are shown in fig. 3 as compared with the "standard" sample $(0.1<y<0.9)$. Since at small $y$ there are no low- $x$ data, only asymmetries for $x>0.032$ are plotted. A clear increase of the Sivers asymmetry is visible for the low- $y$ data. This strong effect could be associated with the smaller values of $Q^{2}$ and/or with the smaller values of $W$, the invariant mass of the hadron system. The standard sample corresponds to $W>5 \mathrm{GeV}$, while $W$ values are as low as $\sim 3 \mathrm{GeV}$ in the range $0.05<y<0.1$. While a $Q^{2}$ dependence is expected and has been calculated [7], no dependence on $y$ (nor or $W$ ) is expected.

\section{The spin averaged Azimuthal Asymmetries}

The amplitudes of the $\cos \phi, \cos 2 \phi$ and $\sin \phi$ modulations extracted separately for positive 


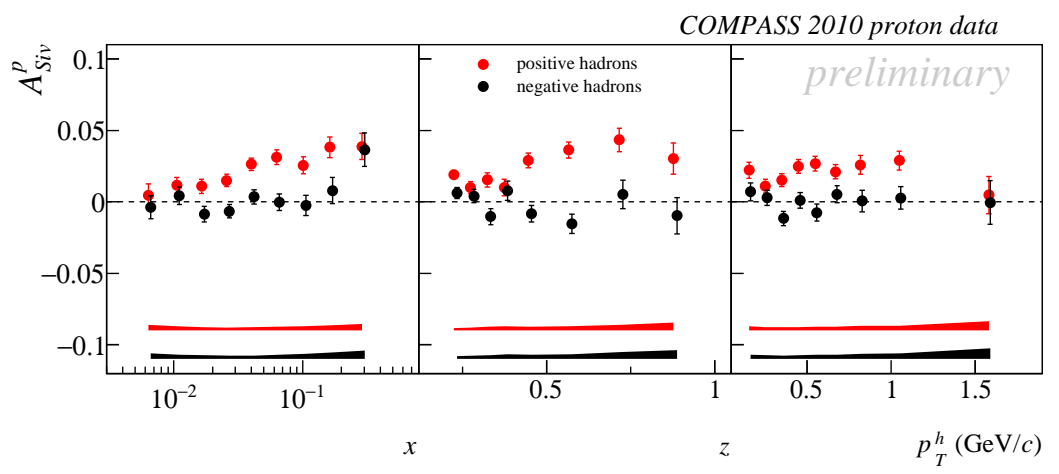

Figure 2: Sivers asymmetry as function of $x, z$ and $P_{T}^{h}$, for positive and negative hadrons.

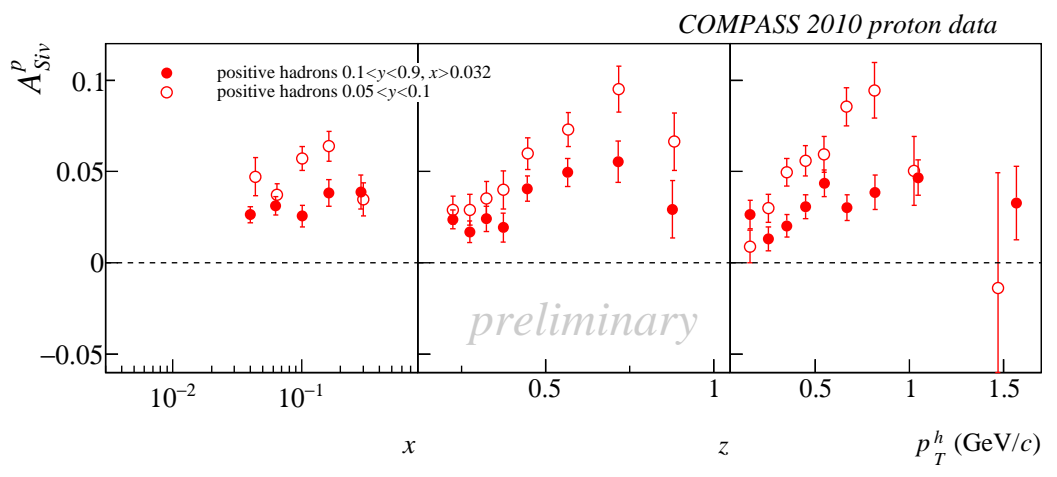

Figure 3: Sivers asymmetry for positive hadrons as function of $x, z$ and $P_{T}^{h}$, for $x>0.032$. The full points refer to the $0.1<y<0.9$ sample, the open points to the $0.05<y<0.1$ sample.

and negative hadrons have been measured from the 2004 deuteron data [10]. The corresponding asymmetries were extracted binning alternatively the data in $x, z$ and $P_{T}^{h}$. The hadron azimuthal distributions calculated from the real data were corrected for the acceptance effects using Monte Carlo simulations of the apparatus. All the possible effects, the geometrical acceptance, the triggers' and detectors' inefficiencies and the interaction of the particles with the material of the spectrometer, were simulated. The same program as for the real data was used for the tracks reconstruction. The results for $\cos \phi$ are shown in fig. . Large asymmetries and a very strong dependence in $z$ are found. To investigate in more details the interesting kinematical dependencies shown in fig. $₫$, more studies have been done, and a multi dimensional analysis has been performed binning simultaneously the data in the 3 variables $x, z$ and $P_{T}^{h}$.

The first COMPASS results of the asymmetries extracted in each 3-dimensional bin are shown in fig. 5 for the $\cos \phi$ modulation, and in fig. 6 for the $\cos 2 \phi$ modulation. The systematical error has been evaluated to be roughly twice the statistical one.

\section{Conclusion}

The azimuthal asymmetries on the transversely polarized target obtained from the 2010 COMPASS proton data are very precise and provide an excellent input to new global fits. The kinemati- 


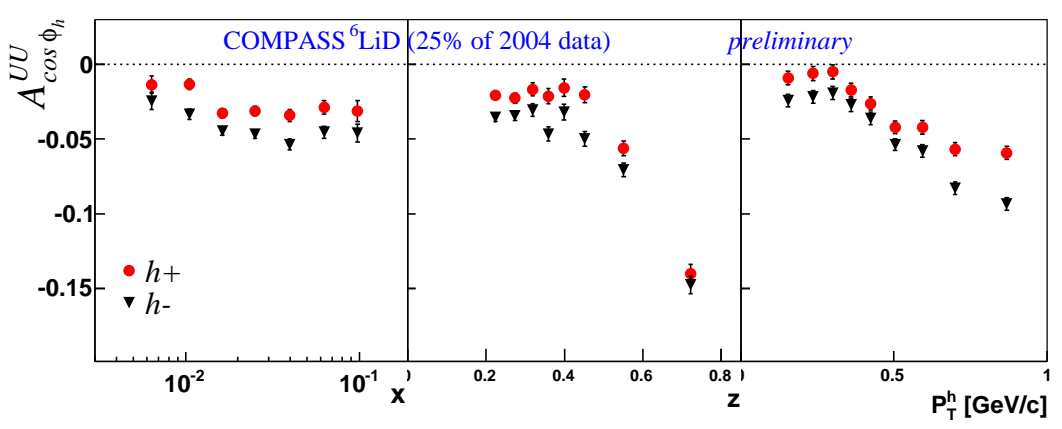

Figure 4: The $\cos \phi$ spin averaged asymmetries as functions of $x, z$ and $P_{T}^{h}$, for the positive hadrons (red points) and negative hadrons (black points).

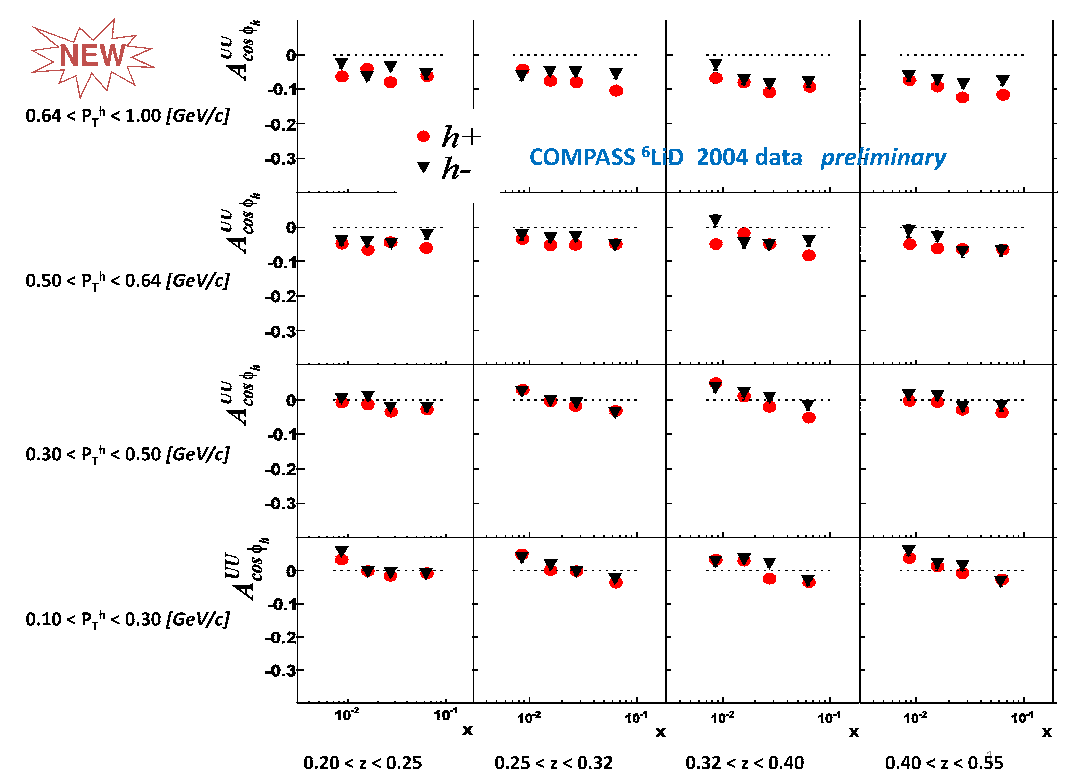

Figure 5: The $\cos \phi$ spin averaged asymmetries as functions of $x$, extracted in different bins of $z$ and $P_{T}^{h}$, for the positive hadrons (red points) and negative hadrons (black triangles).

cal dependencies of the transversity and the Sivers function can be better understood, in particular their $Q^{2}$ evolution. More input for flavor separation will come from the measurement of the asymmetries for identified hadrons, which analysis is presently ongoing. Multi dimensional analysis already started for the extraction of the spin averaged azimuthal asymmetries, which are related to the intrinsic transverse momentum of the quark and its correlation with spin. The new results extracted on deuteron are also very interesting and more will come when also the asymmetries extracted in the large $z$ region, in which the signal seems to be stronger, will be finalized. The study of the azimuthal asymmetries extracted from the huge amount of SIDIS data collected by COMPASS on polarized target is not yet at its end and more refined results containing more detailed information on the nucleon structure will come. Moreover a SIDIS measurement on the unpolarized $\mathrm{LH}_{2}$ target is foreseen in parallel to the DVCS measurement starting from 2015. 


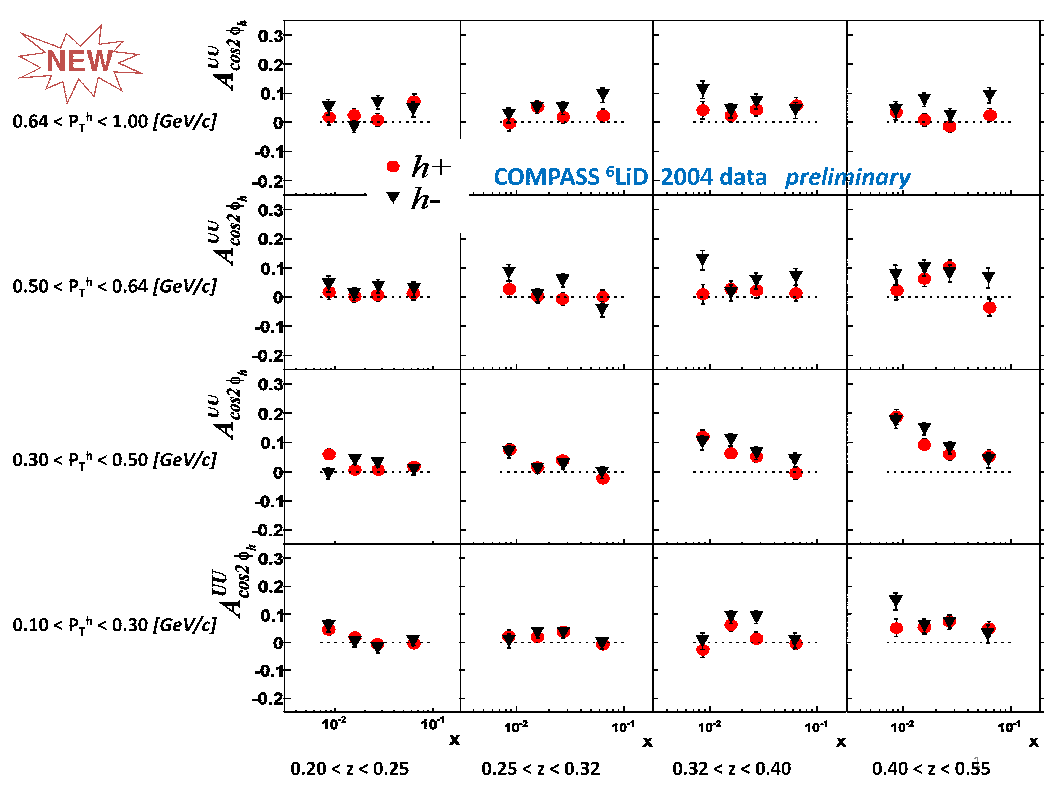

Figure 6: The $\cos 2 \phi$ spin averaged asymmetries as functions of $x$, extracted in different bins of $z$ and $P_{T}^{h}$, for the positive hadrons (red points) and negative hadrons (black triangles).

\section{References}

[1] P. Abbon et al. (The COMPASS Collaboration), The COMPASS Experiment at CERN Nucl. Instr. and Meth. A577 (2007) 455 [hep-ex/ 0703049 ]

[2] J.C. Collins Fragmentation of transversely polarized quarks probed in transverse momentum distributions, Nucl. Phys. B396 (1993) 161 [hep-ph/9208213]

[3] D.W. Sivers, Single Spin Production Asymmetries from the Hard Scattering of Point-Like Constituents Phys. Rev D41 (1990) 83

[4] E.S. Ageev et al. (The COMPASS Collaboration), A new measurement of the Collins and Sivers asymmetries on a transversely polarized deuteron target Nucl. Phys. $\mathbf{B 7 6 5}$ (2007) 31 [hep-ex/0610068]

[5] M.G. Alekseev et al. (The COMPASS Collaboration), Measurement of the Collins and Sivers asymmetries on transversely polarized protons Phys. Lett. B692 (2010) 240 [hep-ex/1005 . 5609]

[6] F. Bradamante, for the COMPASS Collaboration, COMPASS results: new measurements of the Collins and Sivers asymmetries, in proceedings of Transversity 2011, Nuovo Cimento Vol. 35 C, N. 2 [hep-ex/1111.0869v1]

[7] S. Mert Aybat, A. Prokudin and Ted C. Rogers, Calculation of TMD Evolution for Transverse Single Spin Asymmetry Measurements, hep-ph/1112.4423

[8] S.J. Brodsky, D.S. Hwang, I. Schmidt, Final state interactions and single spin asymmetries in semi inclusive deep inelastic scattering, Phys. Lett B530 (2002) 99 [hep-ph/0201296]

[9] J.C. Collins Leading twist single transverse-spin asymmetries: Drell-Yan and deep inelastic scattering, Phys. Lett. B536 (2002) 43 [hep-ph/ 0204004$]$

[10] G. Sbrizzai, Azimuthal asymmetries in the unpolarized SIDIS cross section at COMPASS, in proceedings of SPIN2010 Conference, J. Phys.: Conf. Ser. 295012043 